\title{
Identification of a novel homozygous frameshift mutation in SLC29A3 gene in a case with H syndrome from Iran
}

\author{
Rita Bagheriana, Farideh Yousefipourb, Houriyeh Sadat Mousavic, Fatemeh Saffarid, Elham HajiShafiehac, Samin \\ Nesbat Mohammadic, Hadi Mousakhanie, Seyed Mohammad Fathif, Amirhossein Mehrtashg, Fatemeh Masomi Verkic,
} Donghyun Leeh, Abolfazl Heidaric,i,

a Pediatric Gastroenterology, Children Growth Research center, Qazvin University of Medical Sciences, Qazvin, Iran b National Institute of Genetic Engineering and Biotechnology, Tehran, Iran c Sana Medical Genetics Laboratory, Qazvin, Iran d Pediatric Endocrinology, Children Growth Research Center, Qazvin University of Medical Sciences, Qazvin, Iran e Pediatric Hematology and Oncology, Children Growth Research Center, Qazvin University of Medical Sciences, Qazvin, Iran f Pediatric Clinical Immunology, Children Growth Research Center, Qazvin University of Medical Sciences, Qazvin, Iran g Ronash Medical Genetics Laboratory, Tehran, Iran h The Hospital for Sick Children, University of Toronto, Canada i Refrence Laboratory of Qazvin Medical University, Qazvin, Iran

\begin{abstract}
A B S T R A C T
$\mathrm{H}$ syndrome is a rare monogenic autosomal recessive disease with characteristic cutaneous findings and multisystem involvement. The aim of this study is to present an Iranian patient with $\mathrm{H}$ syndrome and to describe a novel frameshift mutation in SLC29A3 gene. The patient was diagnosed with a few small areas of hyperpigmentation and accompanying hypertrichosis in the lumbar area of her back. Her clinical phenotypes included short stature, hepatosplenomegaly, facial widespread bilateral telangiectatic lesions, bilateral hypertrophy of the parotid gland, upper extremity flexion contracture, elevated inflammatory markers (ESR, CRP) and diabetes mellitus. The identification of a novel homozygous frameshift mutation (c.307_308delTT, p.F103Ter) in SLC29A3 gene, together with the characteristic clinical manifestations of $\mathrm{H}$ syndrome, provided accurate diagnosis for this patient. () 2019 Elsevier Masson SAS. All rights reserved.
\end{abstract}

